\title{
EAHP 2011 Congress: Prize-winning posters
}

Congratulations to the winners of the poster awards which were submitted for the Vienna congress, judged on aspects like originality, scientific quality and practical applicability.

The full posters as displayed during the congress and which provide more detail, can be seen on the subsequent pages.

\section{$1^{\text {st }}$ PRIZE}

\section{DEVELOPMENT OF A WEB-BASED} ANTIMICROBIAL RESOURCE TO IMPROVE ANTIMICROBIAL PRESCRIBING Ñ A TWO YEAR REVIEW

P. Howard, J.A.T. Sandoe, A. Olusoga, K. Warburton, J. Dunne Pharmacy, Leeds Teaching Hospitals NHS Trust, Leeds, United; Kingdom; Microbiology, Leeds Teaching Hospitals NHS Trust, Leeds, United Kingdom; Leeds Health Pathways, Leeds Teaching Hospitals NHS Trust, Leeds, United Kingdom

Background Our Teaching Hospitals were poorly performing for Clostridium difficile infection (CDI) and MRSA bacteraemia, despite 'CDIfriendly' antibiotics used in patients $>80$ years.

Purpose The project was to build a web-based infection-management resource to improve antimicrobial prescribing by providing:

- evidence-based, peer-reviewed guidelines

- educational resource

- audit resources with results

- decision support including calculators

Material and Methods Templates were developed for each guideline development team of a clinician, microbiologist and pharmacist. Each guideline includes algorithms, investigations, empiric and directed therapy (including special populations), oral switch, duration, specialist referral criteria, references, review dates, and evidence levels. Drafts are peer reviewed for 4 weeks where they are endorsed without changes or with minor revision, or need a major revision. Guidelines are updated based on comments, and repeat peer-review if necessary. Once ratified, uploaded to Antimicrobial Resource website.

\section{Results}

- 104 guidelines developed; average 73 draft views; 7 comments per draft (3 had second peerreview)

- >7000 hits/month

- Antimicrobial prevalence decreased from 35\% to $\sim 25 \%$

- CDI decreased from 80 to 20 cases/month

Discussion Many Trusts use pocket-sized guidelines which go out of date. Our pathways focus on diagnosis and investigations, with antimicrobials if necessary. Development processes promotes ownership and subsequent usage. Feedback mechanisms ensure continual update. Less patients are on antimicrobials or develop CDI. Changes in prescribing may have contributed.

Conclusion Web-based, evidenced-based, peerreviewed antimicrobial guidelines are an effective method to support prescribers in their diagnosis and treatment of infection. Links to resources such as eBNF, eMC and dose calculators improve patient safety. Feedback processes with regular update ensure that guidelines are always up-to-date. Guidelines designed and delivered in this manner promote usage, and when combined with other elements of antimicrobial stewardship, is associated with a decrease in the prevalence of antimicrobial usage and reductions in some HCAIs.

No conflict of interest. 


\title{
Development of a Web-Based Antimicrobial Resource to Improve Antimicrobial Prescribing - A Two Year Review
}

\author{
$\underline{\text { P. Howard }}^{1}$, J.A.T. Sandoe ${ }^{2}$, A. Olusoga ${ }^{1}$, K. Warburton ${ }^{3}$, J. Dunne ${ }^{3}$ \\ ${ }^{1}$ Pharmacy, ${ }^{2}$ Microbiology, \& ${ }^{3}$ Leeds Health Pathways - \\ The Leeds Teaching Hospitals NHS Trust, Leeds, United Kingdom
}

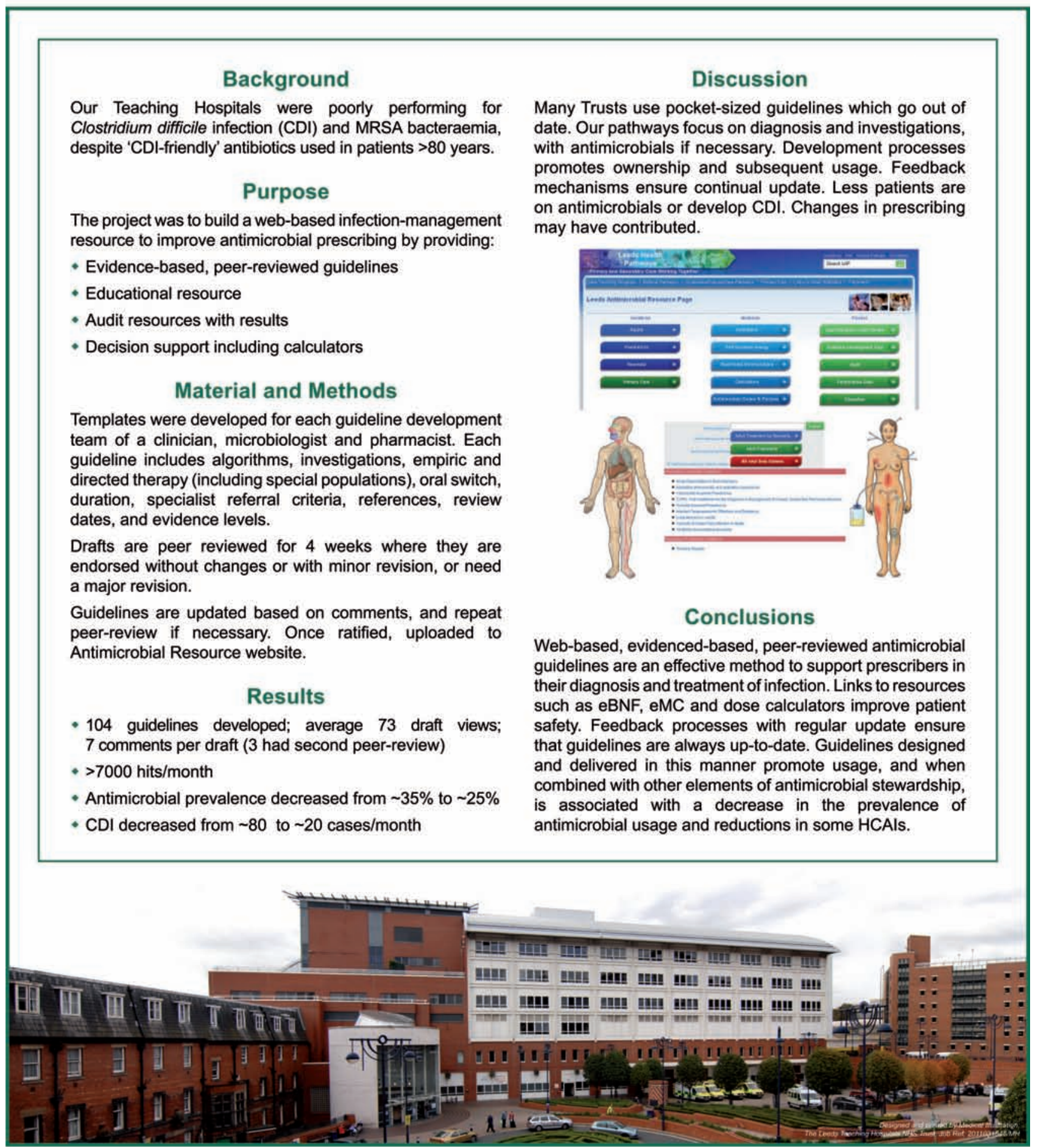




\section{$2^{\text {nd }}$ PRIZE}

\section{WHAT CAN HOSPITAL PHARMACISTS DO FOR PATIENT SAFETY WHEN ENCOUNTERING POTENTIALLY COUNTERFEIT MEDICINES PURCHASED BY PATIENTS OVER THE INTERNET}

A. Fittler, E. Lankó, B. Brachmann, L. Botz University of Pécs Medical School, Department of Pharmaceutics and Central Clinical Pharmacy, Pécs, Hungary; University of Pécs Medical School, First Department of Internal Medicine, Pécs, Hungary

Background The popularity and the number of internationally operated illegal online drugstores that are selling medications without prescriptions or deliver products with unknown origins are rising. As Europeans are spending billions of Euros on the illicit medicines market the chances of accidental overdose, drug interactions and toxicity is increasing.

Purpose To estimate the significance of the problem in Hungary and to define adequate methods to assess the quality and potential danger of drugs sold online.

Material and Methods The attitude of more than 500 patients regarding purchasing drugs online was evaluated in our survey implemented in hospital $(\mathrm{n}=107)$ and community pharmacy $(\mathrm{n}=434)$ setting.

A comprehensive methodology was set up by our institution which allows general and professional quality assessment by:

- Standardised ranking method of online drugstores
- Documentation, evaluation of distribution process

- Identification of microbiologic contamination

- Measurement of physical properties by pharmacotechnology methods

- Chemical analysis of active substance

Results Our results show that nearly $5 \%$ of the respondents of the questionnaire have ordered drugs or dietary supplements online and about same amount of people are considering this option in the near future. 163 online pharmacies were evaluated and followed for 28 months. Less than $7 \%$ of the sites require prior medical prescription and 38\% do not exist after two years. Out of the thirteen medications (paracetamol, sildenafil, tramadol) test ordered, 11 arrived (85\%). Main components were identified (HPLC, spectrophotometry) in all samples. Compared to original authorized medications, higher chemical contamination was observed, indicating lower quality ingredients. The increased microbiological contamination and the higher standard deviation of pharmacotechnology parameters suggest poor quality control of production.

Conclusion Our observations not only draw the attention of hospital pharmacists to illegal online drugstores and counterfeit medicines but also suggest a comprehensive methodology for professional pharmaceutical quality assessment of medication ordered online.

No conflict of interest 


\title{
What can hospital pharmacists do for patient safety when encountering potentially counterfeit medicines purchased by patients over the internet
}

\author{
Department of Pharmaceutics and Central Clinical Pharmacy University of Pécs, Pécs, Hungary
}

Buying medicine online: protecting patients is a new challenge for pharmacists

The popularity and the number of internationally operated illegal online drugstores that are selling medications without prescriptions or deliver products with unknown origins are rising. As Europeans are spending billions of Euros on the illicit medicines market the chances of accidental overdose, drug interactions and toxicity is increasing. The national and international regulation of these sites is currently an unsolved problem world-wide.

The purpose of our work is to assess the current situation of ordering of online medicines in Hungary, to survey the attitude of patients regarding online drug purchase, to define adequate methods to determine the quality and potential danger of drugs sold online and to recommend useful tools for pharmacists to improve patient safety.

$$
\text { People are willing to buy their medicine online, but are not fully aware of the risks }
$$

The attitude of 856 patients regarding purchasing drugs online was evaluated in our survey, which was implemented in hospital $(n=422)$ and community pharmacy ( $n=434)$ setting in ten Hungarian cities. Our results show that $7,3 \%$ of the respondents of the questionnaire have ordered drugs or dietary supplements online and $5,6 \%$ are considering this option in the near future. These numbers are expected to increase, because every sixth respondent (16,3\%) would buy such products from foreign websites if lower prices are offered. Majority of the patients $(78,8 \%)$ don't know anything about the quality of medicines purchased online, only ten percent think that medicines in conventional community pharmacies have higher quality.

$$
\text { Effective methods can be used for the evaluation of quality of medicines purchased over the internet }
$$

A comprehensive methodology was set up which allows general and professional quality assessment of websites and medicines purchased online. Each tool focuses on critical indicators of professionalism, quality and patient safety. The essentials, the applied techniques and the results of each tool are described here below.
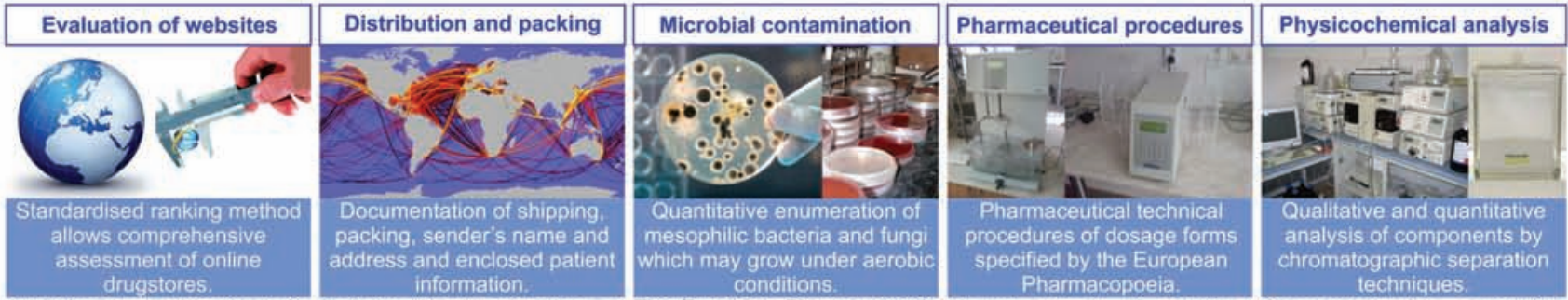

A brief tool was developed for By inspecting the arrived parcel The European Pharmacopoeia Measurement of physical Chromatographic separation the evaluation of internet several signs can indicate illegal determines the microbiological properties and examination of techniques and absorption pharmacies and the potential activity or the absence of quality of pharmaceutical dosage form according to spectrophotometry allow the indicators of damaged patient professionalism. The following preparations. Medications for monographs of the European measurement of the active safety. The questionnaire questions should bechecked: oral and rectal administration Pharmacopoeia should be substance and the identification consists of four set of questions: 1. Did the package arrive?

A: Identification of the supplier 2. Does the sender's name, and criteria:

B: Userfriendliness and address match with the

appearance

C: Appraisal of payment and 3. Does the actual drug match delivery conditions

D: Guarantees for patient with the name, strength and dosage form stated earlier? safety

4. Was it properly stored during Necessity of prescription from a shipment?

doctor, completeness of product 5. Does it have appropriate description and patient infor- packaging?

mation leaflet, quality of online 6 . Is the medicine intact, medical questionnaire must be damaged? surveyed. By the calculation of the 8 . Is the patientinformation weighted total score the risk of leafletincluded?

1. Total viable aerobic count must be measured.

2. Not more than $10^{3}$ bacteria per gram can be present.

3. Not more than $10^{2}$ fungiper

gram can be present.

4. Escherichia colimust be absent.

5. Tests for specified micro-

Required methods of solid prep-1. Identification of substances arations intended for oral ad- by typical spectrum,

ministration (tablets, capsules): 2 . Thin layer chromatography is 1. Organoleptic and microscopic examination,

2. Uniformity of content

3. Uniformity of mass,

4. Dissolution test, a ruginosa, Staphylococcus determine errors and the product that can be deficiencies the manufacturing compared to original authorized inappropriate medication use, Each piece of information given nation indicates inappropriate gathered that can indicate the counterfeits can be identified if identity and credit card theft, or by the supplier on the website manufacture, packing, storage lack of professionalism and the quantity of active ingredien the likelihood of buying must be set against the and distribution of medicines. All quality control. Discrepancies differs significantly, or impurities counterfeit and substandard properties of the delivered can be linked to counterfeit and high standard deviations indicate use of substandard raw medications can be estimated. product. drugs. suggest poor quality products. materials for manufacturing.

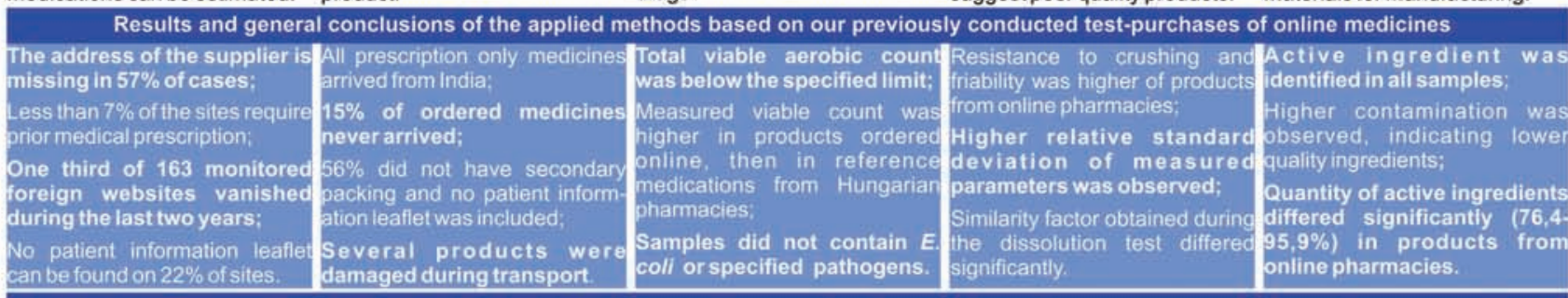

What hospital pharmacist can do for patient safety

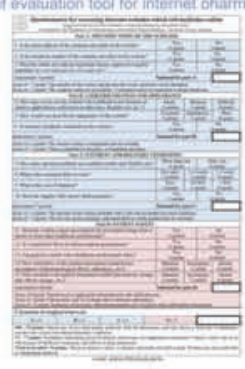

Pharmacists and hospital pharmacists can play an essential role in:
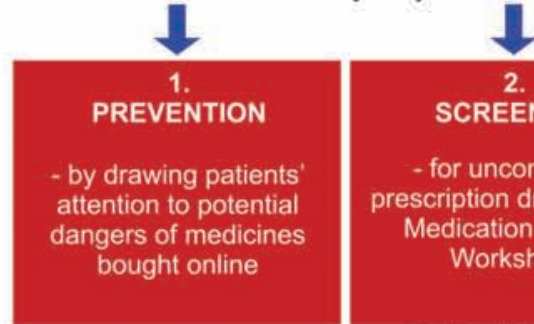
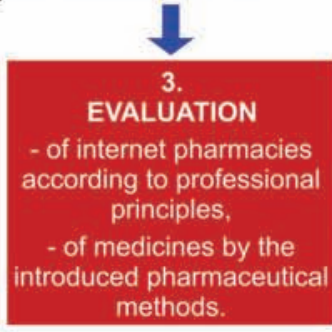


\section{$3^{\text {rd }}$ PRIZE}

\section{MERCAPTOPURINE SUSPENSION $10 \mathrm{MG} / \mathrm{ML}$}

\section{D.J. Postma, H.W.G. Wagenaar Royal Dutch Pharmaceutical Society, Den Haag, Den Haag, The Netherlands}

Background Internationally, the WHO emphasizes the importance of developing new paediatric medicines. In the Netherlands, this importance is generally recognized. When industry does not provide suitable preparations, formulations should be developed for small scale compounding in public or hospital pharmacies. To this aim, the Royal Dutch Association of Pharmacists (KNMP) cooperates closely with the Special Interest Group on Paediatric Medicine of the Dutch Association of Hospital Pharmacists (NVZA). One of the developed formulations is Mercaptopurine suspension $10 \mathrm{mg} / \mathrm{ml}$.

Purpose Mercaptopurine is practically insoluble in water. This means that an oral, liquid preparation with mercaptopurine is only feasible as a suspension. Literature1 suggests that ascorbic acid should be added as anti-oxidant, to protect mercaptopurine for oxidation. The need for an anti-oxidant is doubted, because the mercaptopurine will be merely suspended and is not dissolved. A stability study has been performed on two formulations, with and without ascorbic acid. Aim of the study is to find a formulation that yields a stable suspension for at least 6 months.

Material and methods Several batches of mercaptopurine suspension $10 \mathrm{mg} / \mathrm{ml}$ were prepared in a standard suspension base, with and without ascorbic acid. The batches, packed in PET-bottles, were kept at $25{ }^{\circ} \mathrm{C}$ for 6 months. Samples were taken at 0, 1, 3, 6 and 9 months, and were analysed for appearance, $\mathrm{pH}$, viscosity, related substances and content of mercaptopurine. For the assay a stability-indicating HPLC-method was used, based on the Ph.Eur. monograph for the active ingredient.

Results and conclusion All suspensions show stability for at least 6 months, regarding the content of mercaptopurine. The addition of ascorbic acid has no additional value. On the contrary, ascorbic acid causes a slight colouration of the suspension, while the viscosity decreases in time. This is unwanted in regard to the physical stability. It is concluded that a formulation without ascorbic acid yields the most stable suspension.

\section{Literature}

1. Am J Health Syst Pharm 65(2008):441-7.

No conflict of interest 


\title{
Mercaptopurine suspension $10 \mathrm{mg} / \mathrm{ml}$
}

\author{
D.J. Postma ${ }^{1}$, H.W.G. Wagenaar ${ }^{1}$ \\ ${ }^{1}$ Royal Dutch Pharmacists Association (KNMP), Alexanderstraat 11, 2514 JL, Den Haag. The Netherlands. T 0031-70-3737284; F 0031-70-4274801; E d.j.postma@knmp.n]
}

\section{Objectives}

Internationally, the WHO emphasizes the importance of developing new paediatric medicines. In the Netherlands, this importance is generally recognized. When industry does not provide suitable preparations for children, formulations should be developed for small scale compounding in public or hospital pharmacies. To this aim, the Royal Dutch Pharmacists Association (KNMP) cooperates closely with the Special Interest Group on Paediatric Medicine of the Dutch Association of Hospital Pharmacists (NVZA). One of the developed formulations is Mercaptopurine suspension $10 \mathrm{mg} / \mathrm{ml}$.

Mercaptopurine is practically insoluble in water. This means that an oral, liquid preparation with mercaptopurine is only feasible as a suspension. Literature ${ }^{1}$ suggests that ascorbic acid should be added as anti-oxidant, to protect mercaptopurine against oxidation. The need for an anti-oxidant is doubtful, because the mercaptopurine will be merely suspended and is not dissolved. Aim of the study is to find a formulation that yields a stable suspension for at least 6 months.

\section{Methods and study design}

Several batches of mercaptopurine suspension $10 \mathrm{mg} / \mathrm{ml}$ were prepared in a standard suspension base, with and without ascorbic acid in a concentration of $1 \mathrm{mg} / \mathrm{ml}$. The composition of the batches is shown in table 1 . The batches, packed in PET-bottles, were kept at $25^{\circ} \mathrm{C}$ for 6 months. Samples were taken at 0, 1, 3, 6 and 9 months, and were analysed for appearance, $\mathrm{pH}$, viscosity, related substances and content of mercaptopurine. For the assay a stability-indicating HPLC-method was used, based on the Ph.Eur. monograph for the active ingredient.
Table 1: Composition of Mercaptopurine suspension

\begin{tabular}{lcl} 
Mercaptopurinum & 1 & 9 \\
\hline Acidum citricum monohydricum cryst & 74 & $\mathrm{mg}$ \\
\hline Aluminii et magnesii silicas colloidale & 991 & $\mathrm{mg}$ \\
Carmellosum natricum M & 991 & $\mathrm{mg}$ \\
Methylis parahydroxybenzoas & 74 & $\mathrm{mg}$ \\
Sirupus simplex & 26 & $\mathrm{~g}$ \\
Aqua purificata & 77,87 & $\mathrm{~g}$
\end{tabular}

\section{Discussion}

The results of the study show that addition of ascorbic acid has no additional value regarding the mercaptopurine content. However, ascorbic acid causes a slight colouration of the suspension, while the viscosity decreases in time. Although no effect on the homogeneity is observed, a decrease in viscosity is considered as an unwanted effect in regard to the physical stability of the suspension.

\section{Results}

All suspensions show satisfying stability for at least 6 months at $25^{\circ} \mathrm{C}$, regarding the content of mercaptopurine (figure 1 ). Only the viscosity of a suspension with ascorbic acid is considerably lower than a suspension without ascorbic acid, and furthermore, viscosity decreases in time (figure 2). The decrease does not have a measurable effect on the homogeneity of the suspension.

\section{Conclusion}

It is concluded that a formulation without ascorbic acid yields a stable suspension, which can be stored for 6 months at $25^{\circ} \mathrm{C}$.

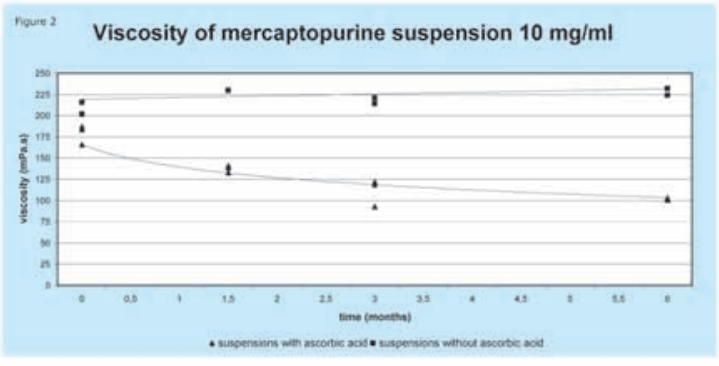

\section{Literature}

1. Am J Health Syst Pharm 65(2008): 441-7.

\section{Acknowledgements}

We would like to thank the Erasmus University Hospital, Rotterdam, for kindly providing us the batches for the stability study. 